\title{
Reference-less Quality Estimation of Text Simplification Systems
}

\author{
Louis Martin \\ Facebook AI Research \& Inria \\ louismartin@fb.com
}

\author{
Antoine Bordes \\ Facebook AI Research \\ abordes@fb.com
}

\author{
Samuel Humeau \\ Facebook AI Research \\ samuelhumeau@fb.com
Éric de La Clergerie Inria \\ eric.de_la_clergerie@inria.fr
}

\author{
Pierre-Emmanuel Mazaré \\ Facebook AI Research \\ pem@fb.com
}

\author{
Benoît Sagot \\ Inria \\ benoit.sagot@inria.fr
}

\begin{abstract}
The evaluation of text simplification (TS) systems remains an open challenge. As the task has common points with machine translation (MT), TS is often evaluated using MT metrics such as BLEU. However, such metrics require high quality reference data, which is rarely available for TS. TS has the advantage over MT of being a monolingual task, which allows for direct comparisons to be made between the simplified text and its original version. In this paper, we compare multiple approaches to reference-less quality estimation of sentence-level text simplification systems, based on the dataset used for the QATS 2016 shared task. We distinguish three different dimensions: grammaticality, meaning preservation and simplicity. We show that $n$-gram-based MT metrics such as BLEU and METEOR correlate the most with human judgment of grammaticality and meaning preservation, whereas simplicity is best evaluated by basic length-based metrics.
\end{abstract}

\section{Introduction}

Text simplification (hereafter TS) has received increasing interest by the scientific community in recent years. It aims at producing a simpler version of a source text that is both easier to read and to understand, thus improving the accessibility of text for people suffering from a range of disabilities such as aphasia (Carroll et al., 1998) or dyslexia (Rello et al., 2013), as well as for second language learners (Xia et al., 2016) and people with low literacy (Watanabe et al., 2009). This topic has been researched for a variety of languages such as English (Zhu et al., 2010; Wubben et al., 2012; Narayan and Gardent, 2014; Xu et al., 2015), French (Brouwers et al., 2014), Spanish (Saggion et al., 2011), Portuguese (Specia, 2010), Italian (Brunato et al., 2015) and Japanese (Goto et al., 2015). ${ }^{1}$

One of the main challenges in TS is finding an adequate automatic evaluation metric, which is necessary to avoid the time-consuming human evaluation. Any TS evaluation metric should take into account three properties expected from the output of a TS system, namely:

- Grammaticality: how grammatically correct is the TS system output?

- Meaning preservation: how well is the meaning of the source sentence preserved in the TS system output?

- Simplicity: how simple is the TS system output? ${ }^{2}$

TS is often reduced to a sentence-level problem, whereby one sentence is transformed into a simpler version containing one or more sentences. In this paper, we shall make use of the terms source (sentence) and (TS system) output to respectively denote a sentence given as an input to a TS system and the simplified, single or multi-sentence output produced by the system.

TS, seen as a sentence-level problem, is often viewed as a monolingual variant of (sentencelevel) MT. The standard approach to automatic TS evaluation is therefore to view the task as a translation problem and to use machine translation (MT)

\footnotetext{
${ }^{1}$ Note that text simplification has also been used as a preprocessing step for other natural language processing tasks such as machine translation (Chandrasekar et al., 1996) and semantic role labelling (Vickrey and Koller, 2008).

${ }^{2}$ There is no unique way to define the notion of simplicity in this context. Previous works often rely on the intuition of human annotators to evaluate the level of simplicity of a TS system output.
} 
evaluation metrics such as BLEU (Papineni et al., 2002). However, MT evaluation metrics rely on the existence of parallel corpora of source sentences and manually produced reference translations, which are available on a large scale for many language pairs (Tiedemann, 2012). TS datasets are less numerous and smaller. Moreover, they are often automatically extracted from comparable corpora rather than strictly parallel corpora, which results in noisier reference data. For example, the PWKP dataset (Zhu et al., 2010) consists of 100,000 sentences from the English Wikipedia automatically aligned with sentences from the Simple English Wikipedia based on term-based similarity metrics. It has been shown by $\mathrm{Xu}$ et al. (2015) that many of PWKP's "simplified" sentences are in fact not simpler or even not related to their corresponding source sentence. Even if better quality corpora such as Newsela do exist (Xu et al., 2015), they are costly to create, often of limited size, and not necessarily open-access.

This creates a challenge for the use of referencebased MT metrics for TS evaluation. However, TS has the advantage of being a monolingual translation-like task, the source being in the same language as the output. This allows for new, nonconventional ways to use MT evaluation metrics, namely by using them to compare the output of a TS system with the source sentence, thus avoiding the need for reference data. However, such an evaluation method can only capture at most two of the three above-mentioned dimensions, namely meaning preservation and, to a lesser extent, grammaticality.

Previous works on reference-less TS evaluation include Štajner et al. (2014), who compare the behaviour of six different MT metrics when used between the source sentence and the corresponding simplified output. They evaluate these metrics with respect to meaning preservation and grammaticality. We extend their work in two directions. Firstly, we extend the comparison to include the degree of simplicity achieved by the system. Secondly, we compare additional features, including those used by Štajner et al. (2016a), both individually, as elementary metrics, and within multi-feature metrics. To our knowledge, no previous work has provided as thorough a comparison across such a wide range and combination of features for the reference-less evaluation of TS.

First we review available text simplification evaluation methods and traditional quality estimation features. We then present the QATS shared task and the associated dataset, which we use for our experiments. Finally we compare all methods in a reference-less setting and analyze the results.

\section{Existing evaluation methods}

\subsection{Using MT metrics to compare the output and a reference}

TS can be considered as a monolingual translation task. As a result, MT metrics such as BLEU (Papineni et al., 2002), which compare the output of an MT system to a reference translation, have been extensively used for TS (Narayan and Gardent, 2014; Štajner et al., 2015; Xu et al., 2016). Other successful MT metrics include TER (Snover et al., 2009), ROUGE (Lin, 2004) and METEOR (Banerjee and Lavie, 2005), but they have not gained much traction in the TS literature.

These metrics rely on good quality references, something which is often not available in TS, as discussed by Xu et al. (2015). Moreover, Štajner et al. (2015) and Sulem et al. (2018a) showed that using BLEU to compare the system output with a reference is not a good way to perform TS evaluation, even when good quality references are available. This is especially true when the TS system produces more than one sentence for a single source sentence.

\subsection{Using MT metrics to compare the output and the source sentence}

As mentioned in the Introduction, the fact that TS is a monolingual task means that MT metrics can also be used to compare a system output with its corresponding source sentence, thus avoiding the need for reference data. Following this idea, Štajner et al. (2014) found encouraging correlations between 6 widely used MT metrics and human assessments of grammaticality and meaning preservation. However MT metrics are not relevant for the evaluation of simplicity, which is why they did not take this dimension into account. Xu et al. (2016) also explored the idea of comparing the TS system output with its corresponding source sentence, but their metric, SARI, also requires to compare the output with a reference. In fact, this metric is designed to take advantage of more than one reference. It can be applied when only one reference is available for each source sentence, but its results are better when multiple ref- 
erences are available.

Attempts to perform Quality Estimation on the output of TS systems, without using references, include the 2016 Quality Assessment for Text Simplification (QATS) shared task (Štajner et al., 2016b), to which we shall come back in section 3 . Sulem et al. (2018b) introduce another approach, named SAMSA. The idea is to evaluate the structural simplicity of a TS system output given the corresponding source sentence. SAMSA is maximized when the simplified text is a sequence of short and simple sentences, each accounting for one semantic event in the original sentence. It relies on an in-depth analysis of the source sentence and the corresponding output, based on a semantic parser and a word aligner. A drawback of this approach is that good quality semantic parsers are only available for a handful of languages. The intuition that sentence splitting is an important sub-task for producing simplified text motivated Narayan et al. (2017) to organize the Split and Rephrase shared task, which was dedicated to this problem.

\subsection{Other metrics}

One can also estimate the quality of a TS system output based on simple features extracted from it.

For instance, the QUEST framework for quality estimation in MT gives a number of useful baseline features for evaluating an output sentence (Specia et al., 2013). These features range from simple statistics, such as the number of words in the sentence, to more sophisticated features, such as the probability of the sentence according to a language model. Several teams who participated in the QATS shared task used metrics based on this framework, namely SMH (Štajner et al., 2016a), UoLGP (Rios and Sharoff, 2015) and UoW (Béchara et al., 2015).

Readability metrics such as Flesch-Kincaid Grade Level (FKGL) and Flesch Reading Ease (FRE) (Kincaid et al., 1975) have been extensively used for evaluating simplicity. These two metrics, which were shown experimentally to give good results, are linear combinations of the number of words per sentence and the number of syllables per word, using carefully adjusted weights.

\section{Methodology}

Our goal is to compare a large number of ways to perform TS evaluation without a reference. To

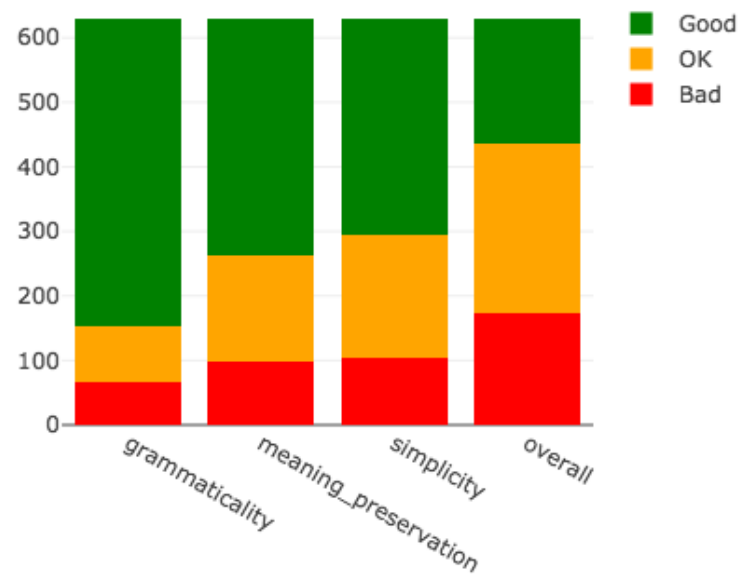

Figure 1: Label repartition on the QATS Shared task

this end, we use the dataset provided in the QATS shared task. We first compare the behaviour of elementary metrics, which range from commonly used metrics such as BLEU to basic metrics based on a single low-level feature such as sentence length. We then compare the effect of aggregating these elementary metrics into more complex ones and compare our results with the state of the art, based on the QATS shared task data and results.

\subsection{The QATS shared task}

The data from the QATS shared task (Štajner et al., 2016b) consists of a collection of 631 pairs of english sentences composed of a source sentence extracted from an online corpus and a simplified version thereof, which can contain one or more sentences. This collection is split into a training set (505 sentence pairs) and a test set (126 sentence pairs). Simplified versions were produced automatically using one of several TS systems trained by the shared task organizers. Human annotators labelled each sentence pair using one of the three labels Good, $\mathrm{OK}$ and $\mathrm{Bad}$ on each of the three dimensions: grammaticality, meaning preservation and simplicity ${ }^{3}$. An overall quality label was then automatically assigned to each sentence pair based on its three manually assigned labels using a method detailed in (Štajner et al., 2016b). Distribution of the labels and examples are presented in FIGURE 1 and TABLE 1.

The goal of the shared task is, for each sentence in the test set, to either produce a label (Good, OK,

\footnotetext{
${ }^{3}$ We were not able to find detailed information about the annotation process. In particular, we do not know whether each sentence was annotated only once or whether multiple annotations were produced, followed by an adjudication step.
} 


\begin{tabular}{|c|c|c|c|c|c|c|}
\hline \multirow{2}{*}{ Version } & \multirow{2}{*}{ Sentence } & \multicolumn{4}{|c|}{ Aspect } & \multirow{2}{*}{ Modification } \\
\hline & & $\mathrm{G}$ & M & S & $\mathrm{O}$ & \\
\hline Original & $\begin{array}{l}\text { All three were arrested in the Toome area and have been taken } \\
\text { to the Serious Crime Suite at Antrim police station. }\end{array}$ & good & good & good & good & syntactic \\
\hline Simple & $\begin{array}{l}\text { All three were arrested in the Toome area. All three have been } \\
\text { taken to the Serious Crime Suite at Antrim police station. }\end{array}$ & & & & & \\
\hline Original & $\begin{array}{l}\text { For years the former Bosnia Serb army commander Ratko } \\
\text { Mladic had evaded capture and was one of the worlds most } \\
\text { wanted men, but his time on the run finally ended last year } \\
\text { when he was arrested near Belgrade. }\end{array}$ & good & bad & ok & bad & content reduction \\
\hline Simple & $\begin{array}{l}\text { For years the former Bosnia Serb army commander Ratko } \\
\text { Mladic had evaded capture. }\end{array}$ & & & & & \\
\hline Original & $\begin{array}{l}\text { Madrid was occupied by French troops during the Napoleonic } \\
\text { Wars, and Napoleons brother Joseph was installed on the } \\
\text { throne. }\end{array}$ & good & good & good & good & lexical \\
\hline Simple & $\begin{array}{l}\text { Madrid was occupied by French troops during the Napoleonic } \\
\text { Wars, and Napoleons brother Joseph was put on the throne. }\end{array}$ & & & & & \\
\hline Original & $\begin{array}{l}\text { Keeping articles with potential encourages editors, especially } \\
\text { unregistered users, to be bold and improve the article to allow it } \\
\text { to evolve over time. }\end{array}$ & bad & bad & ok & bad & dropping \\
\hline Simple & $\begin{array}{l}\text { Keeping articles with potential editors, especially unregistered } \\
\text { users, to be bold and improve the article to allow it to evolve } \\
\text { over time. }\end{array}$ & & & & & \\
\hline
\end{tabular}

Table 1: Examples from the training dataset of QATS. Differences between the original and the simplified version are presented in bold. This table is adapted from Štajner et al. (2016b).

$B a d)$ or a raw score estimating the overall quality of the simplification for each of the three dimensions. Raw score predictions are evaluated using the Pearson correlation with the ground truth labels, while actual label prediction are evaluated using the weighted F1-score. The shared task is described in further details on the QATS website ${ }^{4}$.

\subsection{Features}

In our experiments, we compared about 60 elementary metrics, which can be organised as follows:

\section{- MT metrics}

- BLEU, ROUGE, METEOR, TERp

- Variants of BLEU: BLEU_1gram, BLEU_2gram, BLEU_3gram, BLEU_4gram and seven smoothing methods ${ }^{5}$ from NLTK (Bird and Loper, 2004).

- Intermediate components of TERp inspired by (Štajner et al., 2016a): e.g. number of insertions, deletions, shifts...

\footnotetext{
${ }^{4}$ http://qats2016.github. io/shared.html

${ }^{5}$ https://www.nltk.org/api/nltk. translate.html\#nltk.translate.bleu_ score. SmoothingFunction
}

- Readability metrics and other sentence-level features: FKGL and FRE, numbers of words, characters, syllables...

- Metrics based on the baseline QuEst features (17 features) (Specia et al., 2013), such as statistics on the number of words, word lengths, language model probability and $n$ gram frequency.

- Metrics based on other features: frequency table position, concreteness as extracted from Brysbaert et al.'s 2014 list, language model probability of words using a convolutional sequence to sequence model from (Gehring et al., 2017), comparison methods using pretrained fastText word embeddings (Mikolov et al., 2018) or Skip-thought sentence embeddings (Kiros et al., 2015).

TABLE 2 lists 30 of the elementary metrics that we compared, which are those that we found to correlate the most with human judgments on one or more of the three dimensions (grammaticality, meaning preservation, simplicity).

\subsection{Experimental setup}

Evaluation of elementary metrics We rank all features by comparing their behaviour with human 
judgments on the training set. We first compute for each elementary metric the Pearson correlation between its results and the manually assigned labels for each of the three dimensions. We then rank our elementary metrics according to the absolute value of the Pearson correlation. ${ }^{6}$

Training and evaluation of a combined metric We use our elementary metrics as features to train classifiers on the training set, and evaluate their performance on the test set. We therefore scale them and reduce the dimensionality with a 25-component $\mathrm{PCA}^{7}$, then train several regression algorithms ${ }^{8}$ and classification algorithms ${ }^{9}$ using scikit-learn (Pedregosa et al., 2011). For each dimension, we keep the two models performing best on the test set and add them in the leaderboard of the QATS shared task (TABLE 4), naming them with the name of the regression algorithm they were built with.

\section{Results}

\subsection{Comparing elementary metrics}

FIGURE 3 ranks all elementary metrics given their absolute Pearson correlation on each of the three dimensions.

Grammaticality $N$-gram based MT metrics have the highest correlation with human grammaticality judgments. METEOR seems to be the best, probably because of its robustness to synonymy, followed by smoothed BLEU (BLEUSmoothed in 2). This indicates that relevant grammaticality information can be derived from the source sentence. We were expecting that information contained in a language model would help achieving better results (AvgLMProbsOutput), but MT metrics correlate better with human judgments. We deduce that the grammaticality information contained in the source is more specific and more helpful for evaluation than what is learned by the language model.

\footnotetext{
${ }^{6} \mathrm{We}$ will release our code on github.

${ }^{7}$ We used PCA instead of feature selection because it performed better on the validation set. The number of component was tuned on the validation set as well.

${ }^{8}$ Regressors: Linear regression, Lasso, Ridge, Linear SVR (SVM regressor), Adaboost regressor, Gradient boosting regressor and Random forest regressor.

${ }^{9}$ Classifiers: Logistic regression, MLP classifier (with L2 penalty, alpha=1), SVC (linear SVM classifier), Knearsest neighbors classifier ( $k=3)$, Adaboost classifier, Gradient boosting classifier and Random forest classifier.
}

Meaning preservation It is not surprising that meaning preservation is best evaluated using MT metrics that compare the source sentence to the output sentence, with in particular smoothed BLEU, BLEU_3gram and METEOR. Very simple features such as the percentage of words in common between source and output also rank high. Surprisingly, word embedding comparison methods do not perform as well for meaning preservation, even when using word alignment.

Simplicity Methods that give the best results are the most straightforward for assessing simplicity, namely word, character and syllable counts in the output, averaged over the number of output sentences. These simple features even outperform the traditional, more complex metrics FKGL and FRE. As could be expected, we find that metrics with the highest correlation to human simplicity judgments only take the output into account. Exceptions are the NBSourceWords and NBSourcePunct features. Indeed, if the source sentence has a lot of words and punctuation, and is therefore likely to be particularly complex, then the output will most likely be less simple as well. We also expected word concreteness ratings and position in the frequency table to be good indicators of simplicity, but it does not seem to be the case here. Structural simplicity might simply be more important than such more sophisticated components of the human intuition of simple text.

Discussion Even if counting the number of words or comparing $n$-grams are good proxies for the simplification quality, they are still very superficial features and might miss some deeper and more complex information. Moreover the fact that grammaticality and meaning preservation are best evaluated using $n$-gram-based comparison metrics might bias the TS models towards copying the source sentence and applying fewer modifications.

Syntactic parsing or language modelling might capture more insightful grammatical information and allow for more flexibility in the simplification model. Regarding meaning preservation, semantic analysis or paraphrase detection models would also be good candidates for a deeper analysis.

Warning note We should be careful when interpreting these results as the QATS dataset is relatively small. We compute confidence intervals on our results, and find them to be non-negligible, yet without putting our general observations into 


\begin{tabular}{ll}
\hline Short name & Description \\
\hline NBSourcePunct & Number of punctuation tokens in source (QUEST) \\
NBSourceWords & Number of source words (QUEST) \\
NBOutputPunct & Number of punctuation tokens in output (QUEST) \\
TypeTokenRatio & Type token ratio (QUEST) \\
TERp_Del & Number of deletions (TERp component) \\
TERp_NumEr & Number of total errors (TERp component) \\
TERp_Sub & Number of substitutions (TERp component) \\
TERp & TERp MT metric \\
BLEU_1gram & BLEU MT metric with unigrams only \\
BLEU_2gram & BLEU MT metric up to bigrams \\
BLEU_3gram & BLEU MT metric up to trigrams \\
BLEU_4gram & BLEU MT metric up to 4-grams \\
METEOR & METEOR MT metric \\
ROUGE & ROUGE summarization metric \\
BLEUSmoothed & BLEU MT metric with smoothing (method 7 from nltk) \\
AvgCosineSim & Cosine similarity between source and output pre-trained word embeddings \\
NBOutputChars & Number of characters in the output \\
NBOutputCharsPerSent & Average number of characters per sentence in the output \\
NBOutputSyllables & Number of syllables in the output \\
NBOutputSyllablesPerSent & Average number of syllables per sentence in the output \\
NBOutputWords & Number of words in the output \\
NBOutputWordsPerSent & Average number of words per sentence in the output \\
AvgLMProbsOutput & Average log-probabilities of output words (Language Model) \\
MinLMProbsOutput & Minimum log-probability of output words (Language Model) \\
MaxPosInFreqTable & Maximum position of output words in the frequency table \\
AvgConcreteness & Average word concreteness Brysbaert et al.'s 2014 concreteness list \\
OutputFKGL & Flesch-Kincaid Grade Level \\
OutputFRE & Flesch Reading Ease \\
WordsInCommon & Percentage of words in common between source and Output \\
\hline
\end{tabular}

Table 2: Brief description of 30 of our most relevant elementary metrics

\begin{tabular}{|c|c|c|c|c|c|c|c|c|}
\hline $\begin{array}{l}\text { Grammaticality } \\
\text { Short name }\end{array}$ & Train $\downarrow$ & Test & $\begin{array}{l}\text { Meaning Preservation } \\
\text { Short name }\end{array}$ & Train $\downarrow$ & Test & $\begin{array}{l}\text { Simplicity } \\
\text { Short name }\end{array}$ & Train $\downarrow$ & Test \\
\hline Best QATS team & & 0.48 & Best QATS team & & 0.59 & Best QATS team & & 0.38 \\
\hline METEOR & 0.36 & 0.39 & BLEUSmoothed & 0.59 & 0.52 & NBOutputCharsPerSent & -0.52 & -0.45 \\
\hline BLEUSmoothed & 0.33 & 0.34 & BLEU_3gram & 0.57 & 0.52 & NBOutputSyllablesPerSent & -0.52 & -0.49 \\
\hline BLEU_4gram & 0.32 & 0.34 & METEOR & 0.57 & 0.58 & NBOutputWordsPerSent & -0.51 & -0.39 \\
\hline BLEU_3gram & 0.31 & 0.34 & BLEU_2gram & 0.57 & 0.52 & NBOutputChars & -0.48 & -0.37 \\
\hline TERp_NumEr & -0.30 & -0.31 & BLEU_4gram & 0.57 & 0.51 & NBOutputWords & -0.47 & -0.29 \\
\hline BLEU_2gram & 0.30 & 0.34 & WordsInCommon & 0.55 & 0.50 & NBOutputSyllables & -0.46 & -0.42 \\
\hline TERp & -0.30 & -0.32 & BLEU_1gram & 0.55 & 0.52 & NBOutputPunt & -0.42 & -0.31 \\
\hline ROUGE & 0.29 & 0.29 & ROUGE & 0.55 & 0.47 & NBSourceWords & -0.38 & -0.21 \\
\hline AvgLMProbsOutput & 0.28 & 0.34 & TERp & -0.54 & -0.48 & outputFKGL & -0.36 & -0.37 \\
\hline BLEU_1gram & 0.27 & 0.33 & TERp_NumEr & -0.53 & -0.49 & NBSourcePunct & -0.34 & -0.18 \\
\hline WordsInCommon & 0.27 & 0.30 & TERp_Del & -0.50 & -0.52 & okenRatio & -0.22 & -0.04 \\
\hline TERp_Del & -0.27 & -0.35 & AvgCosineSim & 0.44 & 0.34 & AvgConcreteness & 0.21 & 0.32 \\
\hline NBSourceWords & -0.25 & -0.07 & AvgLMProbsOutput & 0.39 & 0.36 & MaxPosInFreqTable & -0.18 & 0.03 \\
\hline AvgCosineSim & 0.23 & 0.25 & AvgConcreteness & -0.28 & -0.06 & MinLMProbsOutput & 0.17 & 0.15 \\
\hline MinLMProbsOutput & 0.11 & -0.07 & NBSourceWords & -0.28 & -0.13 & OutputFRE & 0.16 & 0.27 \\
\hline
\end{tabular}

Table 3: Pearson correlation with human judgments of elementary metrics ranked by absolute value on training set (15 best metrics for each dimension). 
question. For instance, METEOR, which performs best on grammaticality, has a $95 \%$ confidence interval of $0.36 \pm 0.08$ on the training set. These results are therefore preliminary and should be validated on other datasets.

\subsection{Combination of all features with trained models}

We also combine all elementary metrics and train an evaluation models for each of the three dimensions. TABLE 4a presents our two best regressors in validation for each of the dimensions and TABLE $4 b$ for classifiers.

Pearson correlation for regressors (raw scoring) Combining the features does not bring a clear advantage over the elementary metrics METEOR and NBOutputSyllablesPerSent. Indeed our best models score respectively on grammaticality, meaning preservation and simplicity: 0.33 (Lasso), 0.58 (Ridge) and 0.49 (Ridge) versus 0.39 (METEOR), 0.58 (METEOR) and 0.49 (NBOutputSyllablesPerSent).

It is surprising to us that the aggregation of multiple elementary features would score worse than the features themselves. However, we observe a strong discrepancy between the scores obtained on the train and test set, as illustrated by TABLE 3 . We also observed very large confidence intervals in terms of Pearson correlation. For instance our lasso model scores $0.33 \pm 0.17$ on the test set for grammaticality. This should observe caution when interpreting Pearson scores on QATS.

F1-score for classifiers (assigning labels) On the classification task, our models seem to score best for meaning preservation, simplicity and overall, and third for grammaticality. This seems to confirm the importance of considering a large ensemble of elementary features including lengthbased metrics to evaluate simplicity.

\section{Conclusion}

Finding accurate ways to evaluate text simplification (TS) without the need for reference data is a key challenge for TS, both for exploring new approaches and for optimizing current models, in particular those relying on unsupervised, often MT-inspired models.

We explore multiple reference-less quality evaluation methods for automatic TS systems, based on data from the 2016 QATS shared task. We rely on the three key dimensions of the quality of a TS system: grammaticality, meaning preservation and simplicity.

Our results show that grammaticality and meaning preservation are best assessed using $n$-grambased MT metrics evaluated between the output and the source sentence. In particular, METEOR and smoothed BLEU achieve the highest correlation with human judgments. These approaches even outperform metrics that make an extensive use of external data, such as language models. This shows that a lot of useful information can be obtained from the source sentence itself.

Regarding simplicity, we observe that counting the number of characters, syllables and words provides the best results. In other words, given the currently available metrics, the length of a sentence seems to remain the best available proxy for its simplicity.

However, given the small size of the QATS dataset and the high variance observed in our experiments, these results must be taken with a pinch of salt and will need to be confirmed on a larger dataset. Creating a larger annotated dataset as well as averaging multiple human annotations for each pair of sentences would help reducing the variance of the experiments and confirming our findings.

In future work, we shall explore richer and more complex features extracted using syntactic and semantic analyzers, such as those used by the SAMSA metric, and paraphrase detection models.

Finally, it remains to be understood how we can optimize the trade-off between grammaticality, meaning preservation and simplicity, in order to build the best possible comprehensive TS metric in terms of correlation with human judgments. Unsurprisingly, optimizing one of these dimensions often leads to lower results on other dimensions (Schwarzer and Kauchak, 2018). For instance, the best way to guarantee grammaticality and meaning preservation is to leave the source sentence unchanged, thus resulting in no simplification at all. Improving TS systems will require better global TS evaluation metrics. This is especially true when considering that TS is in fact a multiply defined task, as there are many different ways of simplifying a text, depending on the different categories of people and applications at whom TS is aimed. 


\begin{tabular}{llll}
\hline Grammaticality & Meaning Preservation & Simplicity & Overall \\
\hline 0.482 OSVCML1 & 0.588 IIT-Meteor & 0.487 Ridge & 0.423 Ridge \\
0.384 METEOR & 0.585 OSVCML & 0.456 LinearSVR & 0.423 LinearRegression \\
0.344 BLEU & 0.575 Ridge & 0.382 OSVCML1 & 0.343 OSVCML2 \\
0.340 OSVCML & 0.573 OSVCML2 & 0.376 OSVCML2 & 0.334 OSVCML \\
0.327 Lasso & 0.555 Lasso & 0.339 OSVCML & 0.232 SimpleNets-RNN2 \\
0.323 TER & 0.533 BLEU & 0.320 SimpleNets-MLP & 0.230 OSVCML1 \\
0.308 SimpleNets-MLP & 0.527 METEOR & 0.307 SimpleNets-RNN3 & 0.205 UoLGP-emb \\
0.308 WER & 0.513 TER & 0.240 SimpleNets-RNN2 & 0.198 SimpleNets-MLP \\
0.256 UoLGP-emb & 0.495 WER & 0.123 UoLGP-combo & 0.196 METEOR \\
0.256 UoLGP-combo & 0.482 OSVCML1 & 0.120 UoLGP-emb & 0.189 UoLGP-combo \\
0.208 UoLGP-quest & 0.465 SimpleNets-MLP & 0.086 UoLGP-quest & 0.144 UoLGP-quest \\
0.118 GradientBoostingRegressor & 0.285 UoLGP-quest & 0.052 IIT-S & 0.130 TER \\
0.064 SimpleNets-RNN3 & 0.262 SimpleNets-RNN2 & -0.169 METEOR & 0.112 SimpleNets-RNN3 \\
0.056 SimpleNets-RNN2 & 0.262 SimpleNets-RNN3 & -0.242 TER & 0.111 WER \\
& 0.250 UoLGP-combo & -0.260 WER & 0.107 BLEU \\
& 0.188 UoLGP-emb & -0.267 BLEU & \\
\hline
\end{tabular}

(a) Pearson correlation for regressors (raw scoring)

\begin{tabular}{|c|c|c|c|}
\hline Grammaticality & Meaning Preservation & Simplicity & Overall \\
\hline 71.84 SMH-RandForest & 70.14 SVC & $61.60 \mathrm{SVC}$ & 49.61 LogisticRegression \\
\hline 71.64 SMH-IBk & 68.07 SMH-Logistic & 56.95 AdaBoostClassifier & 48.57 SMH-RandForest-b \\
\hline 70.43 LogisticRegression & 65.60 MS-RandForest & 56.42 SMH-RandForest-b & $48.20 \mathrm{UoW}$ \\
\hline 69.96 SMH-RandForest-b & 64.40 SMH-RandForest & 53.02 SMH-RandForest & 47.54 SMH-Logistic \\
\hline 69.09 BLEU & 63.74 TER & $51.12 \mathrm{SMH}-\mathrm{IBk}$ & 46.06 SimpleNets-RNN2 \\
\hline 68.82 SimpleNets-MLP & 63.54 SimpleNets-MLP & 49.96 SimpleNets-RNN3 & 45.71 AdaBoostClassifier \\
\hline 68.36 TER & 62.82 BLEU & 49.81 SimpleNets-MLP & 44.50 SMH-RandForest \\
\hline 67.60 GradientBoosting & 62.72 MT-baseline & 48.31 MT-baseline & 40.94 METEOR \\
\hline 67.53 MS-RandForest & 62.69 IIT-Meteor & 47.84 MS-IBk-b & 40.75 SimpleNets-RNN3 \\
\hline 67.50 IIT-LM & 61.71 MS-IBk-b & 47.82 MS-RandForest & 39.85 MS-RandForest \\
\hline 66.79 WER & $61.50 \mathrm{MS}-\mathrm{IBk}$ & 47.47 SimpleNets-RNN2 & 39.80 DeepIndiBow \\
\hline 66.75 MS-RandForest-b & 60.38 GradientBoosting & 43.46 IIT-S & 39.30 IIT-Metrics \\
\hline 65.89 DeepIndiBow & 60.12 METEOR & 42.57 DeepIndiBow & 38.27 MS-IBk \\
\hline 65.89 DeepBow & 59.69 SMH-RandForest-b & 40.92 UoW & 38.16 MS-IBk-b \\
\hline 65.89 MT-baseline & 59.06 WER & 39.68 Majority-class & 38.03 DeepBow \\
\hline 65.89 Majority-class & $58.83 \mathrm{UoW}$ & $38.10 \mathrm{MS}-\mathrm{IBk}$ & 37.49 MT-baseline \\
\hline 65.72 METEOR & 51.29 SimpleNets-RNN2 & 35.58 DeepBow & 34.08 TER \\
\hline 65.50 SimpleNets-RNN2 & $51.00 \mathrm{CLaC}-\mathrm{RF}$ & 34.88 CLaC-RF-0.5 & 34.06 CLaC-0.5 \\
\hline 65.11 SimpleNets-RNN3 & 46.64 SimpleNets-RNN3 & 34.66 CLaC-RF-0.6 & 33.69 SimpleNets-MLP \\
\hline 64.39 CLaC-RF-Perp & 46.30 DeepBow & 34.48 WER & 33.04 IIT-Default \\
\hline 62.00 MS-IBk & 42.53 DeepIndiBow & 34.30 CLaC-RF-0.7 & 32.92 BLEU \\
\hline \multirow{4}{*}{ 46.32 UoW } & 42.51 Majority-class & 33.52 TER & 32.88 CLaC-0.7 \\
\hline & & 33.34 METEOR & 32.20 CLaC-0.6 \\
\hline & & 33.00 BLEU & 31.28 WER \\
\hline & & & 26.53 Majority-class \\
\hline
\end{tabular}

(b) Weighted F1 Score for classifiers (assign the label Good, OK or Bad)

Table 4: QATS leaderboard. Results in bold are our additions to the original leaderboard. We only select the two models that rank highest during cross-validation. 


\section{Acknowledgments}

We would like to thank our anonymous reviewers for their insightful comments.

\section{References}

Satanjeev Banerjee and Alon Lavie. 2005. Meteor: An automatic metric for $\mathrm{mt}$ evaluation with improved correlation with human judgments. In Proceedings of the acl workshop on intrinsic and extrinsic evaluation measures for machine translation and/or summarization, pages 65-72.

Hanna Béchara, Hernani Costa, Shiva Taslimipoor, Rohit Gupta, Constantin Orasan, Gloria Corpas Pastor, and Ruslan Mitkov. 2015. Miniexperts: An svm approach for measuring semantic textual similarity. In Proceedings of the 9th international workshop on semantic evaluation (SemEval 2015), pages 96-101.

Steven Bird and Edward Loper. 2004. Nltk: the natural language toolkit. In Proceedings of the ACL 2004 on Interactive poster and demonstration sessions, page 31 . Association for Computational Linguistics.

Laetitia Brouwers, Delphine Bernhard, Anne-Laure Ligozat, and Thomas François. 2014. Syntactic sentence simplification for french. In Proceedings of the 3rd Workshop on Predicting and Improving Text Readability for Target Reader Populations (PITR), pages 47-56.

Dominique Brunato, Felice Dell'Orletta, Giulia Venturi, and Simonetta Montemagni. 2015. Design and annotation of the first italian corpus for text simplification. In Proceedings of The 9th Linguistic Annotation Workshop, pages 31-41.

Marc Brysbaert, Amy Beth Warriner, and Victor Kuperman. 2014. Concreteness ratings for 40 thousand generally known english word lemmas. Behavior research methods, 46(3):904-911.

John Carroll, Guido Minnen, Yvonne Canning, Siobhan Devlin, and John Tait. 1998. Practical simplification of english newspaper text to assist aphasic readers. In Proceedings of the AAAI-98 Workshop on Integrating Artificial Intelligence and Assistive Technology, pages 7-10.

Raman Chandrasekar, Christine Doran, and Bangalore Srinivas. 1996. Motivations and methods for text simplification. In Proceedings of the 16th conference on Computational linguistics-Volume 2, pages 1041-1044. Association for Computational Linguistics.

Jonas Gehring, Michael Auli, David Grangier, Denis Yarats, and Yann N. Dauphin. 2017. Convolutional sequence to sequence learning. arXiv preprint arXiv:1705.03122.
Isao Goto, Hideki Tanaka, and Tadashi Kumano. 2015. Japanese news simplification: Task design, data set construction, and analysis of simplified text. Proceedings of MT Summit XV, 1:17-31.

J. Peter Kincaid, Robert P Fishburne Jr., Richard L. Rogers, and Brad S. Chissom. 1975. Derivation of new readability formulas (automated readability index, fog count and flesch reading ease formula) for navy enlisted personnel.

Ryan Kiros, Yukun Zhu, Ruslan R Salakhutdinov, Richard Zemel, Raquel Urtasun, Antonio Torralba, and Sanja Fidler. 2015. Skip-thought vectors. In Advances in neural information processing systems, pages 3294-3302.

Chin-Yew Lin. 2004. Rouge: A package for automatic evaluation of summaries. Text Summarization Branches Out.

Tomas Mikolov, Edouard Grave, Piotr Bojanowski, Christian Puhrsch, and Armand Joulin. 2018. Advances in pre-training distributed word representations. In Proceedings of the International Conference on Language Resources and Evaluation (LREC 2018).

Shashi Narayan and Claire Gardent. 2014. Hybrid simplification using deep semantics and machine translation. In Proceedings of the 52nd Annual Meeting of the Association for Computational Linguistics (Volume 1: Long Papers), volume 1, pages 435-445.

Shashi Narayan, Claire Gardent, Shay B Cohen, and Anastasia Shimorina. 2017. Split and rephrase. arXiv preprint arXiv:1707.06971.

Kishore Papineni, Salim Roukos, Todd Ward, and WeiJing Zhu. 2002. Bleu: a method for automatic evaluation of machine translation. In Proceedings of the 40th annual meeting on association for computational linguistics, pages 311-318. Association for Computational Linguistics.

Fabian Pedregosa, Gal Varoquaux, Alexandre Gramfort, Vincent Michel, Bertrand Thirion, Olivier Grisel, Mathieu Blondel, Peter Prettenhofer, Ron Weiss, Vincent Dubourg, Jake Vanderplas, Alexandre Passos, David Cournapeau, Matthieu Brucher, Matthieu Perrot, and douard Duchesnay. 2011. Scikit-learn: Machine Learning in Python. Journal of Machine Learning Research, 12:2825-2830.

Luz Rello, Ricardo Baeza-Yates, Stefan Bott, and Horacio Saggion. 2013. Simplify or help?: text simplification strategies for people with dyslexia. In Proceedings of the 10th International CrossDisciplinary Conference on Web Accessibility, page 15 . ACM.

Miguel Rios and Serge Sharoff. 2015. Large scale translation quality estimation. In The Proceedings of the 1st Deep Machine Translation Workshop. 
Horacio Saggion, Elena Gómez Martínez, Esteban Etayo, Alberto Anula, and Lorena Bourg. 2011. Text simplification in simplext. making text more accessible. Procesamiento del lenguaje natural, 47:341-342.

Max Schwarzer and David Kauchak. 2018. Human evaluation for text simplification: The simplicityadequacy tradeoff.

Matthew G Snover, Nitin Madnani, Bonnie Dorr, and Richard Schwartz. 2009. Ter-plus: paraphrase, semantic, and alignment enhancements to translation edit rate. Machine Translation, 23(2-3):117-127.

Lucia Specia. 2010. Translating from complex to simplified sentences. In International Conference on Computational Processing of the Portuguese Language, pages 30-39. Springer.

Lucia Specia, Kashif Shah, Jose GC Souza, and Trevor Cohn. 2013. Quest-a translation quality estimation framework. In Proceedings of the 51st Annual Meeting of the Association for Computational Linguistics: System Demonstrations, pages 79-84.

Sanja Štajner, Hannah Béchara, and Horacio Saggion. 2015. A deeper exploration of the standard pb-smt approach to text simplification and its evaluation. In Proceedings of the 53rd Annual Meeting of the Association for Computational Linguistics and the 7th International Joint Conference on Natural Language Processing (Volume 2: Short Papers), volume 2, pages 823-828.

Sanja Štajner, Ruslan Mitkov, and Horacio Saggion. 2014. One step closer to automatic evaluation of text simplification systems. In Proceedings of the $3 \mathrm{rd}$ Workshop on Predicting and Improving Text Readability for Target Reader Populations (PITR), pages $1-10$.

Sanja Štajner, Maja Popovic, and Hanna Béchara. 2016a. Quality estimation for text simplification. In Proceedings of the QATS Workshop, pages 15-21.

Sanja Štajner, Maja Popovic, Horacio Saggion, Lucia Specia, and Mark Fishel. 2016b. Shared task on quality assessment for text simplification. Training, 218(95):192.

Elior Sulem, Omri Abend, and Ari Rappoport. 2018a. Bleu is not suitable for the evaluation of text simplification.

Elior Sulem, Omri Abend, and Ari Rappoport. 2018b. Semantic structural evaluation for text simplification. In Proceedings of the 2018 Conference of the North American Chapter of the Association for Computational Linguistics: Human Language Technologies, Volume 1 (Long Papers), volume 1, pages 685-696.

Jrg Tiedemann. 2012. Parallel Data, Tools and Interfaces in OPUS. In Proceedings of the 8th International Conference on Language Resources and
Evaluation, LREC'12, pages 2214-2218, Istanbul, Turkey.

David Vickrey and Daphne Koller. 2008. Sentence simplification for semantic role labeling. Proceedings of ACL-08: HLT, pages 344-352.

Willian Massami Watanabe, Arnaldo Candido Junior, Vinícius Rodriguez Uzêda, Renata Pontin de Mattos Fortes, Thiago Alexandre Salgueiro Pardo, and Sandra Maria Aluísio. 2009. Facilita: reading assistance for low-literacy readers. In Proceedings of the 27th ACM international conference on Design of communication, pages 29-36. ACM.

Sander Wubben, Antal Van Den Bosch, and Emiel Krahmer. 2012. Sentence simplification by monolingual machine translation. In Proceedings of the 50th Annual Meeting of the Association for Computational Linguistics: Long Papers-Volume 1, pages 1015-1024. Association for Computational Linguistics.

Menglin Xia, Ekaterina Kochmar, and Ted Briscoe. 2016. Text readability assessment for second language learners. In Proceedings of the 11th Workshop on Innovative Use of NLP for Building Educational Applications, pages 12-22.

Wei Xu, Chris Callison-Burch, and Courtney Napoles. 2015. Problems in current text simplification research: New data can help. Transactions of the Association of Computational Linguistics, 3(1):283297.

Wei Xu, Courtney Napoles, Ellie Pavlick, Quanze Chen, and Chris Callison-Burch. 2016. Optimizing statistical machine translation for text simplification. Transactions of the Association for Computational Linguistics, 4:401-415.

Zhemin Zhu, Delphine Bernhard, and Iryna Gurevych. 2010. A monolingual tree-based translation model for sentence simplification. In Proceedings of the 23 rd international conference on computational linguistics, pages 1353-1361. Association for Computational Linguistics. 